\title{
Latency low handover mechanism considering data traffic lost preventing for proxy mobile IPv6 over WLAN
}

\begin{abstract}
Typical PMIPv6 supports mobility management for the Mobile Host (MH) in localized domains over variant Wireless Local Area Network technologies. The typical PMIPv6 adopted in reactive mode in which break-before-make technique may concern, which results in long disruption latency and inevitable data traffic loss that negatively affects MH's communication performance. This article proposes a proactive latency low handover mechanism, which corresponds to make-before-break technique in order to support MH's seamless and fast roaming in PMIPv6 network. The proposed mechanism proactively performs a pre-registration and pre-access authentication processes tightly together intended for the $\mathrm{MH}$ in advance of a handover situation involved in typical PMIPv6, thereby enabling the $\mathrm{MH}$ to re-configure its interface more quickly after a handover. Consequently, the associated mobility-related signallings along with their latencies are reduced significantly and the continuity of the $\mathrm{MH}$ communication session is granted. Furthermore, an efficient buffering technique with optimized functions is introduced at the MH's anchor mobility entity to prevent data traffic loss and save their transmission cost. Through various simulation evalua- tions via ns-2, we study and analyse different mobility aspects, such as handover latency, data traffic loss, throughput, end-to-end traffic delay, traffic transmission cost and signalling cost, with respect to different traffic sources like CBR-UDP and FTP-TCP. Several experiments were conducted, revealing numerous results that verify the proposed mechanisms' superior performance over existing scheme.
\end{abstract}

Keyword: Network-based; PMIPv6; Handover latency; Traffic loss; Context transfer; Signalling cost 